Check for updates

Cite this: Phys. Chem. Chem. Phys., 2019, 21, 8587

\title{
Insight into the internal structure of amyloid- $\beta$ oligomers by isotope-edited Fourier transform infrared spectroscopy $\dagger$
}

\author{
Cesare M. Baronio, (D) Maurizio Baldassarre (D) and Andreas Barth (D) *
}

\begin{abstract}
The internal structure of amyloid- $\beta$ (A $\beta$ ) oligomers was investigated with isotope-edited Fourier transform infrared spectroscopy. Homo-oligomers of $A \beta_{40}$ and $A \beta_{42}$ were prepared from unlabeled and ${ }^{13} \mathrm{C},{ }^{15} \mathrm{~N}$-labeled monomeric $\mathrm{A} \beta$ and from mixtures of these. For the unlabeled peptides, two main bands were observed in ${ }^{2} \mathrm{H}_{2} \mathrm{O}$ at 1685 and $1622 \mathrm{~cm}^{-1}$ for $A \beta_{40}$ and at 1685 and $1626 \mathrm{~cm}^{-1}$ for $A \beta_{42}$. These band positions indicate that the number of strands per sheet is at least four. The obtained experimental amide I spectra were simulated using a number of structural models (antiparallel $\beta$-sheets, $\beta$-barrels and a dodecamer structure). According to experiments and calculations, the main ${ }^{13} \mathrm{C}$-band shifts down at increasing molar ratio of labeled peptides. This shift occurs when vibrational coupling becomes possible between ${ }^{13} \mathrm{C}$-amide groups in close-by strands. It is small, when intervening ${ }^{12} \mathrm{C}$-strands increase the distance between ${ }^{13} \mathrm{C}$-strands; it is large, when many neighboring strands are labeled. The shift depends on the internal structure of the peptides within the oligomers, i.e. on the building block that each peptide molecule contributes to the $\beta$-sheets of the oligomers. The shift is largest, when individual peptides contribute just a single strand surrounded by strands from other peptide molecules. It is smaller when each molecule forms two or three adjacent strands. As indicated by a comparison between experiment and computation, the number of adjacent $\beta$-strands per peptide molecule is two for $A \beta_{40}$ oligomers and two or more for $A \beta_{42}$ oligomers. Our results are well explained by regular, antiparallel $\beta$-sheets or $\beta$-barrels.
\end{abstract}

Received 5th February 2019,

Accepted 2nd April 2019

DOI: $10.1039 / c 9 c p 00717 b$

rsc.li/pccp

\section{Introduction}

Alzheimer's disease is the most frequent neurodegenerative disease. It is associated with the formation of amyloid plaques in the brain which mainly consist of aggregated amyloid- $\beta$ peptide $(A \beta) . A \beta$ is formed by proteolytic cleavage of the amyloid precursor protein in two main variants which are 40 $\left(\mathrm{A} \beta_{40}\right)$ and $42\left(\mathrm{~A} \beta_{42}\right)$ residues long. Both have a propensity to aggregate, which is stronger for $A \beta_{42}$ due to two additional hydrophobic residues (isoleucine, alanine) at the C-terminus. ${ }^{1-9}$ The aggregation process from monomers to fibrils is complex and involves a large number of oligomeric species, which are thought to contribute considerably to the toxicity of $A \beta .^{4,10-15}$ These oligomers are difficult to investigate because they have not yet been crystallized without modification, they are too small for cryo-electron microscopy and aggregates larger than tetramers are too large for solution NMR. ${ }^{16}$ Nevertheless, several structural

Department of Biochemistry and Biophysics, Stockholm University, Sweden.

E-mail: barth@dbb.su.se

$\dagger$ Electronic supplementary information (ESI) available. See DOI: 10.1039/ c9cp00717b models have been proposed, either for a dimer unit from solution NMR, ${ }^{16}$ from solid state NMR studies ${ }^{17-21}$ or from $\mathrm{X}$-ray crystallography of $\mathrm{A} \beta$ stabilized by a protein scaffold ${ }^{22}$ or by modifying the sequence. ${ }^{23}$ Further information has been obtained from Fourier transform infrared (FTIR) spectroscopy of dried $A \beta_{42}$ oligomers, the spectrum of which resembled most closely that of a bacterial porin with 16 antiparallel $\beta$-strands. ${ }^{24}$ The proposed oligomer structures differ considerably, those that have identified $\beta$-sheet structures - relevant to this work - agree on the existence of antiparallel $\beta$-sheets in the oligomers. Whereas some studies detect them in the C-terminal half $f^{17,22,23}$ or in the C-terminal quarter, ${ }^{19}$ a further study identifies them in the central portion but not in the C-terminal tail. ${ }^{16}$ The discrepancies between the oligomer models call for further investigation, in particular for studies in aqueous solution, considering that most of the present models are based on results in nonaqueous environments.

Infrared spectroscopy is widely used in amyloid research ${ }^{25-28}$ focusing mostly on the absorption of the amide I vibrations. These vibrations consist mainly of the stretching vibration of the carbonyl groups of the peptide backbone. The study of the amide I band is particularly powerful in combination with isotope 
labeling, which can be used to reveal mixing of two different peptides $^{29,30}$ or to study the structure of aggregates. ${ }^{31-36}$

The interpretation of the amide I band profile is enhanced by spectrum calculations. Density function theory can only be applied to small sections of proteins so most approaches focus on a computer-time-efficient description of the amide I vibrations. ${ }^{37-40}$ Each amide I oscillator is assigned an intrinsic frequency, which depends on the electrostatic environment. ${ }^{41-44}$ The individual amide I oscillators couple electrostatically with other amide I oscillators, which is often described by transition dipole coupling. ${ }^{45-47}$ These approximations are insufficient for nearest neighbor interactions which are therefore modeled from density functional theory calculations of small peptides. ${ }^{48-53}$ Conformational dynamics can be accounted for either by statistical variation of the parameters, ${ }^{54-56}$ by averaging spectra of snapshot structures from molecular dynamics simulations ${ }^{57-59}$ or by direct time-domain approaches which also take into account motional narrowing. ${ }^{60-63}$

The relevant model structure for this work are antiparallel $\beta$-sheets. Their amide I vibrations are characterized by a strong coupling between amide groups in adjacent strands. This coupling explains the large splitting of the absorption band into a high and a low wavenumber component. ${ }^{45,64}$ Coupling is less effective when the frequencies of the coupled oscillators are different, for example because the amide groups contain different carbon isotopes. Thus, mixing of different carbon isotopes in a $\beta$-sheet leads to a downshift of the high wavenumber band and an upshift of the low wavenumber band which decreases the splitting between the two bands. ${ }^{65}$

Using a combination of isotope-edited FTIR spectroscopy and spectrum calculation, we have found that mixtures of monomeric $A \beta_{40}$ and $A \beta_{42}$ form mixed hetero-oligomers. ${ }^{65}$ This work is here extended to homo-oligomers of either $A \beta_{40}$ or $A \beta_{42}$. In particular, we describe calculations that aimed to achieve a quantitative agreement with the experiments. Our results show that the internal structure of each peptide in homo-oligomers is such that it contributes at least two adjacent strands to the $\beta$-sheets.

\section{Experimental}

Recombinant, unlabeled and ${ }^{13} \mathrm{C},{ }^{15} \mathrm{~N}$ uniformly labeled $\mathrm{A} \beta_{40}$ and $\mathrm{A} \beta_{42}$ were obtained from AlexoTech (Umeå, Sweden). ${ }^{15} \mathrm{~N}$-Labeling has a very minor effect on the spectrum and will therefore not be mentioned hereafter. Experimental procedures were as described previously. ${ }^{65}$ Briefly, hexafluoroisopropanol(HFIP) treated $\mathrm{A} \beta$ was dissolved in $20 \mathrm{mM} \mathrm{NaO}^{2} \mathrm{H}$ in order to obtain a monomeric peptide solution at a concentration of $200 \mu \mathrm{M}$. Unlabeled and labeled peptides were mixed and $4 \mu \mathrm{L}$ of this solution added to a dry film produced from $4 \mu \mathrm{L}$ of $100 \mathrm{mM}$ sodium phosphate to bring the solution to $\mathrm{p}^{2} \mathrm{H} 7.4$ and initiate aggregation. This preparation was chosen to prevent dilution of the peptide when the $\mathrm{pH}$ was lowered. It produced small-sized oligomers for $A \beta_{40}$ and $A \beta_{42}$ and additional larger aggregates for $A \beta_{42}$ as shown by gel electrophoresis of photo-crosslinked samples. $^{65}$
The peptide solution was placed between two $\mathrm{CaF}_{2}$ windows that were separated by a $50 \mu \mathrm{m}$ plastic spacer. FTIR spectra were recorded approximately $20 \mathrm{~min}$ after initiation of aggregation with a Tensor 37 FTIR spectrometer (Bruker, Germany) that was equipped with a sample shutter. The reference position of the sample shuttle contained a buffer sample that was prepared in the same way as the $A \beta$ samples but without peptide. The spectral resolution was $2 \mathrm{~cm}^{-1}$ and a zero-filling factor of 2 was used. The second derivative spectra were smoothed over 13 data points (approx. $13 \mathrm{~cm}^{-1}$ ) in order to determine the band positions and for the figures in this article. The band position of the ${ }^{13} \mathrm{C}$-band at 0.1 molar ratio of ${ }^{13} \mathrm{C}$-peptides was determined by simultaneous fitting of the absorbance and the second derivative spectrum as described in our previous work. ${ }^{65}$ The band positions at all other ${ }^{13} \mathrm{C}:{ }^{12} \mathrm{C}$ ratios were determined directly from the second derivative spectra.

For an estimation of the secondary structure content, buffer contributions to the absorbance spectrum that were not accounted for by the buffer sample in the reference position of the sample shuttle were subtracted from the sample spectrum as described previously. ${ }^{65}$ Then a straight line between the absorbance values at 1700 and $1600 \mathrm{~cm}^{-1}$ was subtracted from the absorbance spectrum. The data points in the intervals $1710-1700 \mathrm{~cm}^{-1}$ and $1600-1590 \mathrm{~cm}^{-1}$ were set to zero and data points above $1710 \mathrm{~cm}^{-1}$ and below $1590 \mathrm{~cm}^{-1}$ removed. The absorbance spectrum and its second derivative were simultaneously fitted $^{66}$ in the spectral range between 1700 and $1600 \mathrm{~cm}^{-1}$ with the program Kinetics written by Erik Goormaghtigh (Université Libre de Bruxelles) using a scaling factor (weight) of 50 and Savitzky-Golay windows of 9, 13, and 17 points for the second derivative. Six bands were fitted with initial positions at 1683, 1678, 1664, 1645, 1632, and $1624 \mathrm{~cm}^{-1}$ without baseline. These fits produced two bands in the region of the main $\beta$-sheet absorption near $1625 \mathrm{~cm}^{-1}$ at very similar band positions but with widths that differed by a factor of two. The wider band was placed $2 \mathrm{~cm}^{-1}$ higher than the narrower band for both $A \beta_{40}$ and $A \beta_{42}$. The areas obtained were averaged for all three Savitzky-Golay windows used and for three independent experiments for each peptide. They are listed in Table S1 (ESI $\dagger$ ).

Fits with only five bands were also tested for one $A \beta_{40}$ and one $A \beta_{42}$ spectrum. The standard deviation between fit and experimental spectra increased by a factor of more than 2 compared to the 6-band fit. In particular, there was less agreement below $1615 \mathrm{~cm}^{-1}$ for both absorbance spectrum and second derivative spectrum. The band placed initially in the random coil region at $1645 \mathrm{~cm}^{-1}$ moved during the fit into the $1635-1630 \mathrm{~cm}^{-1}$ region, assigned to $\beta$-sheets. This increased the total area assigned to $\beta$-sheets by up to $60 \%$ relative to the area obtained in the 6-band fits. For the 5-band fit to the $A \beta_{42}$ spectrum, the total band area assigned to $\beta$-sheets decreased from $65 \%$ to $50 \%$ when the Savitzky-Golay window increased from 9 to 17 data points. In contrast, the 6-band fits to this spectrum produced a consistent $\beta$-sheet area of $41 \%, 37 \%$, and $42 \%$ for windows of 9,13 , and 17 data points, respectively. Because of the missing but expected band for random coils in the 5-band fits, the more consistent results with the 6-band fits 
and the better agreement between experimental data and 6-band fits, we regard the 6-band fits as a better representation of our experimental data and discuss only these fits hereafter.

\section{Computational methods}

The amide I spectrum was simulated using a Matlab program ${ }^{65,67}$ and model structures with antiparallel $\beta$-sheets. Parallel $\beta$-sheets cannot be used because our approach is based on a matching between experimental and computational spectra (see below). This requires the presence of a distinct high wavenumber $\beta$-sheet band that is not observed in spectra calculated for parallel $\beta$-sheets.

Antiparallel $\beta$-sheets of different sizes were created according to the atomic coordinates suggested by Fraser and MacRae. ${ }^{68}$ Most of the sheets consisted of strands with 10 residues (9 complete amide groups) corresponding to the number of residues in the $\beta$-strands of $A \beta_{40}^{69,70}$ and $A \beta_{42}{ }^{71}$ fibrils. Antiparallel $\beta$-barrels of different sizes were also created, selecting barrel-residing residues from available structures in the protein data bank. The complete list of PDB files and the selected residues for each of these files are listed in the ESI. $\dagger$ Additionally, we considered a complete atomic structure for $A \beta$ oligomers. $^{23}$ The structure is a dodecamer, consisting of four triangular trimers that are tetrahedrically arranged around a central cavity. The structures used are shown in Fig. 1.

The amide I spectrum of our model structures was calculated according to the floating oscillator model ${ }^{37}$ where a protein is regarded as an assembly of individual amide I oscillators that couple via transition dipole coupling. The rest of the structure is not considered. Each oscillator is associated with an intrinsic frequency or wavenumber and a transition

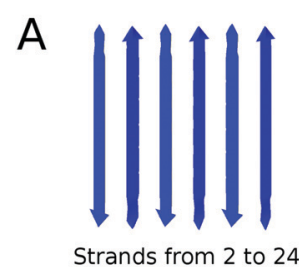

C

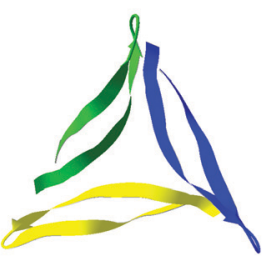

B

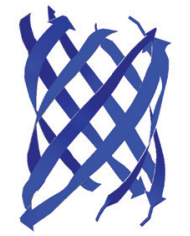

Strands from 8 to 22

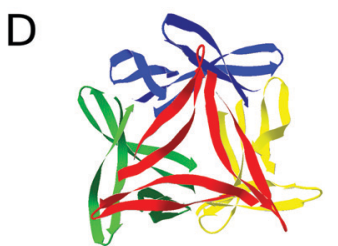

Fig. 1 Representation of the structures used in our calculations. (A) Antiparallel $\beta$-sheets, composed of 2 to 24 strands, (B) $\beta$-barrels, composed of 8 to 22 strands with antiparallel orientation, (C) a trimer unit of the dodecamer structure, (D) the entire dodecamer structure of pdb entry $5 \mathrm{HOW}^{23}$ The structure of each monomer in the dodecamer is a $\beta$-hairpin where two $\beta$-strands are connected by a short loop. In (C) different monomers have different colors. In (D) different trimer units have different colors. The figure was created using Swiss PDB Viewer. ${ }^{72}$ The complete list of models can be found in the ESI. $\dagger$ dipole moment. The latter is proportional to the dipole derivative, which is the change of dipole moment associated with the amide I vibration when the oscillator passes through the equilibrium position. Further effects on the infrared spectrum can be considered as described below. The frequencies and the intensities of the normal modes of vibration are then obtained by a diagonalization of the mass normalized force constant matrix as in our previous work. ${ }^{65,67}$

The diagonal elements of the force constant matrix were calculated from the intrinsic wavenumber, which was assumed to be the same for each unlabeled amide group. The intrinsic wavenumber includes the effects of hydrogen bonding. A constant intrinsic wavenumber for our model structures is therefore equivalent to the assumption of the same hydrogen bonding strength to all amide groups either due to hydrogen bonding to other amide groups or to water. In case of the dodecamer structure we explicitly considered hydrogen-bonding and nearest neighbor effects as in our original version of the program. ${ }^{67}$

The non-diagonal elements of the matrix were obtained using coupling constants from density functional theory calculations for nearest neighbor interactions ${ }^{48,49}$ and transition dipole coupling for all other interactions ${ }^{37}$ with parameters adjusted to match the experimental spectra as described below. The position of the dipole derivative was as suggested by Moore and Krimm. ${ }^{37,73}$ We assumed that the transition dipole moment was the same for each amide group. The angle of the dipole derivative was fixed to $10^{\circ}, 20^{\circ}$ or $30^{\circ}$ degrees with respect to the $\mathrm{C}=\mathrm{O}$ bond, which made the dipole derivative pointing towards the $\mathrm{N}$ atom.

The composition of the force constant matrices and, consequently, the simulation of the absorption spectra depended on the values of the dipole derivative and of the intrinsic wavenumber of the unperturbed amide group. Panel A of Fig. 2 shows that the maximum absorbance and the splitting between high and low wavenumber bands increase when the magnitude of the dipole derivative is increased. Panel B shows that the whole spectrum is shifted towards higher wavenumbers when the intrinsic wavenumber is increased.

The magnitude of the dipole derivative and the intrinsic wavenumber were adjusted for each model structure so that the

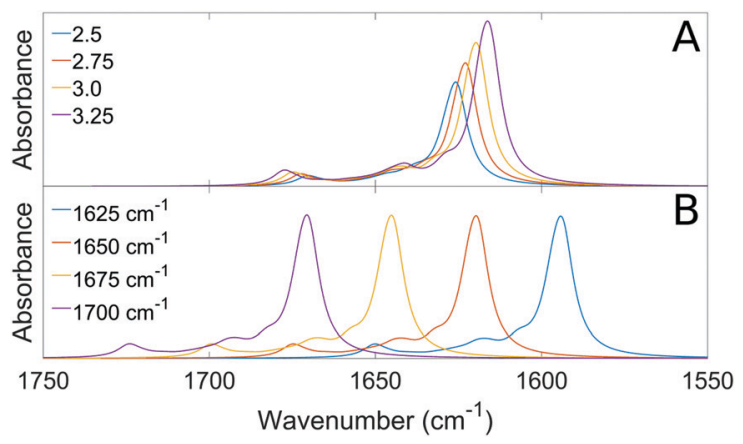

Fig. 2 The calculated absorption spectrum depends on the magnitude of the dipole derivative (panel $\mathrm{A}$ ) and on the intrinsic wavenumber (panel $B$ ). The magnitude of the dipole derivative is given in $D \AA^{-1} \mathrm{amu}^{-1 / 2}$ in panel $A$ and the intrinsic wavenumber in panel $\mathrm{B}$. 


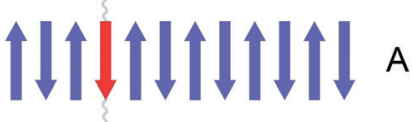

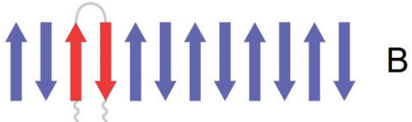

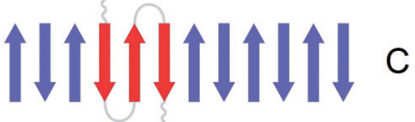
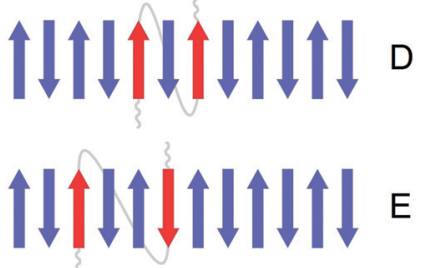

Fig. 3 Illustration of the considered building blocks. A building block is the contribution of an individual peptide (red) to our $\beta$-sheet and $\beta$-barrel model structures. The figure shows the example of an $A \beta$ oligomer with a 12 -stranded antiparallel $\beta$-sheet. Strands from other peptides are shown in blue. (A) Single strand, (B) 2-strand block, (C) 3-strand block, (D) interlaced model 1, (E) interlaced model 2. See text for a detailed description of the building blocks. The non- $\beta$-sheet sections of the peptide with the red $\beta$ strands are schematically shown in grey. The respective sections of other peptides are not shown.

low and high wavenumber band positions of the calculated spectrum matched those in the experimental spectrum of the all-unlabeled sample. The matching between experimental and simulated band positions was performed separately for the $A \beta_{40}$ and the $A \beta_{42}$ spectra. Once the calculation parameters were adjusted for one particular model structure, we used these parameters for the calculation of the spectra for all other ${ }^{13} \mathrm{C}:{ }^{12} \mathrm{C}$ ratios.

We simulated the presence of ${ }^{13} \mathrm{C}$ strands according to the ${ }^{13} \mathrm{C}:{ }^{12} \mathrm{C}$ molar ratios used in the experiments. The strands were labeled according to five different patterns, as shown in Fig. 3, that model the contribution of individual peptides to the structure of the oligomers. This contribution is further on termed building block. All amide groups within one building block are thought to belong to the same peptide molecule and contain the same carbon isotope (with the exception of a $10 \%$ impurity of ${ }^{12} \mathrm{C}$-atoms in ${ }^{13} \mathrm{C}$-peptides, see below). A building block does not comprise the entire peptide molecule and the rest of the peptide is assumed not to participate in $\beta$-sheets. Three building blocks had a different number of adjacent strands from the same peptide. These were:

(A) Single strand: each strand has neighboring strands from other peptides meaning that a given strand might have two adjacent strands with a different carbon isotope.

(B) 2-strand block: each peptide molecule contributes two adjacent strands. Therefore, each strand has one neighboring strand from the same peptide with the same carbon isotope. An example of such a structure is a $\beta$-hairpin where the two antiparallel strands are connected by a loop.
(C) 3-strand block: each peptide molecule contributes three adjacent strands. The middle strand of a 3-strand block has two adjacent strands with the same carbon isotope. An example of such a structure is a 3-stranded $\beta$-sheet where the three strands are connected by two loops. This building block can only be applied to a subset of our model structures where the number of strands is a multiple of 3: $\beta$-sheets with 6,12 and 24 strands; $\beta$-barrels with 12 and 18 strands.

In addition, we considered two types of non-adjacent arrangements:

(D) Interlaced model 1: each peptide molecule contributes two strands which are separated by one strand from a different peptide. This arrangement requires that the number of strands is a multiple of 4 , we used it for our $\beta$-sheets with 4,12 and 24 strands and our $\beta$-barrels with 8,12 and 16 strands.

(E) Interlaced model 2: each peptide molecule contributes two strands that are separated by two strands from two different peptides. For this model the number of strands needs to be a multiple of 6: $\beta$-sheets with 6,12 and 24 strands; $\beta$-barrels with 12 and 18 strands.

For labeled strands, the diagonal elements of the massnormalized force constant matrix were multiplied by 0.94725 . This mass factor was obtained from $A \beta_{40}$ experiments by first matching the simulated and the experimental high and low wavenumber band positions for the ${ }^{12} \mathrm{C}-\mathrm{A} \beta_{40}$ spectrum, as described above, and then simulating the ${ }^{13} \mathrm{C}$-spectrum (considering $10 \%{ }^{12} \mathrm{C}$-impurities in ${ }^{13} \mathrm{C}$-strands, see below) with different mass factors until agreement was obtained between the experimental and simulated band positions of the main $\beta$-sheet band. Our mass factor is close to the ratio of the reduced masses for labeled and unlabeled $\mathrm{C}=\mathrm{O}$ oscillators (0.95604, resulting in a $37 \mathrm{~cm}^{-1}$ down shift from $1650 \mathrm{~cm}^{-1}$ upon ${ }^{13} \mathrm{C}$-labeling). Our mass factor is also close to the ratio of the reduced masses inferred from DFT calculations of trans unlabeled and ${ }^{13} \mathrm{C} \mathrm{N}$-methylacetamide (0.94771, resulting in a $45 \mathrm{~cm}^{-1}$ down shift from $1735 \mathrm{~cm}^{-1}$ upon ${ }^{13} \mathrm{C}$-labeling). ${ }^{74}$

Three situations could occur for the non-diagonal elements. If the non-diagonal element represented an interaction between two unlabeled groups, the force constant remained unchanged. If the non-diagonal element represented an interaction between two labeled groups, the force constant was multiplied by the mass factor (as for the diagonal elements of the labeled groups). If the non-diagonal element represented an interaction between a labeled and an unlabeled group, the force constant was multiplied by the square root of the mass factor.

In order to account for deviations from the used structures (and consequently from the calculated force constants), statistical variations were added to the elements of the force constant matrix. The diagonal elements were subjected to a random variation between $-1 \%$ and $1 \%$ of their original value and the off-diagonal elements between $-10 \%$ and $10 \%$. Furthermore, we considered the $10 \%$ impurity of the labeled peptides used in the experiments: in other words, there is a $10 \%$ chance in our calculations to find unlabeled amide groups inside labeled strands. The presence of the unlabeled amide groups inside labeled strands weakens the coupling within and between 
labeled strands: in the absorbance spectrum the band position of the main band is upshifted and the splitting between high and low wavenumber bands decreases. For example, the splitting for a 6-stranded ${ }^{13} \mathrm{C}$-sheet reduces from $61.0 \mathrm{~cm}^{-1}$ to $57.4 \mathrm{~cm}^{-1}$. As a result of including isotopic impurity, the average splitting for sheets with 4-24 strands and 10 residues/strand and all barrels was $57.5 \mathrm{~cm}^{-1}$, which is very similar to the experimental splitting for ${ }^{13} \mathrm{C}-\mathrm{A} \beta_{40}$, which was $57.7 \mathrm{~cm}^{-1}$ (using parameters adjusted to the ${ }^{12} \mathrm{C}$-spectrum of $\mathrm{A} \beta_{40}$ and a dipole derivative angle of $20^{\circ}$ ).

From the diagonalization of the force constant matrix, we obtained the wavenumbers and the intensities of the normal modes in the amide I region. With this information, the absorbance spectrum was calculated using Gaussian band shapes with a full width at half maximum of $8 \mathrm{~cm}^{-1}$. For a comprehensive statistical sampling of the variations in the force constants, of the isotope composition of the sheets at a given ${ }^{13} \mathrm{C}:{ }^{12} \mathrm{C}$ ratio and of different positions of unlabeled amide groups within labeled strands, 3000 calculations for each ratio were performed and the spectra averaged. The number of 3000 calculations was chosen because it provided a good reproducibility of the spectrum. The calculations were repeated 20 times in order to assess the deviation in the band positions between different simulation runs.

\section{Results}

\section{Experiments}

We measured the infrared spectra of $A \beta$ oligomers that consisted of mixtures of ${ }^{12} \mathrm{C}$ - and ${ }^{13} \mathrm{C}$-isotopomers of either $\mathrm{A} \beta_{40}$ or $A \beta_{42}$. These homo-oligomers are denoted $A \beta_{40}$ oligomers or $\mathrm{A} \beta_{42}$ oligomers in the following. The peptides were mixed as monomers in ${ }^{2} \mathrm{H}_{2} \mathrm{O}$ at alkaline $\mathrm{p}^{2} \mathrm{H}$, then brought to $\mathrm{p}^{2} \mathrm{H} 7.4$ and measured after $20 \mathrm{~min}$, corresponding to the minimal waiting time to accurately purge the spectrometer sample chamber after sample insertion. Fig. 4 shows the second derivatives of the infrared absorption spectra of $A \beta_{40}$ and $A \beta_{42}$ for different ${ }^{13} \mathrm{C}:{ }^{12} \mathrm{C}$-ratios. The minima in second derivatives correspond to the band positions of the component bands in the absorbance spectra. The two major bands are found in

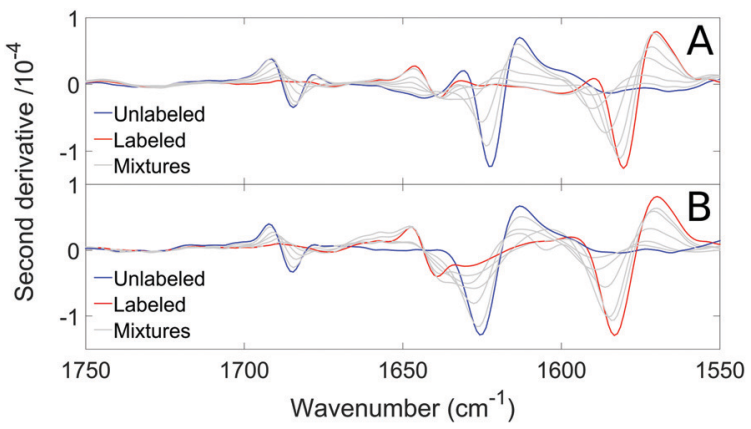

Fig. 4 Spectra of the second derivative of $I R$ absorbance of $(A) A \beta_{40}$ oligomers and (B) $A \beta_{42}$ oligomers at different ${ }^{13} \mathrm{C}:{ }^{12} \mathrm{C}$-ratios. The spectrum of the all-unlabeled sample is blue and that of the all-labeled sample red. Mixtures of unlabeled and labeled $A \beta(9: 1,3: 1,1: 1,1: 3,1: 9)$ are grey.
Table 1 Band positions of the all-unlabeled and all-labeled samples determined from the second derivative using a smoothing range of 13 points

\begin{tabular}{|c|c|c|c|c|}
\hline & \multicolumn{2}{|c|}{$\begin{array}{l}\text { Band position } \pm \text { standard } \\
\text { deviation in } \mathrm{cm}^{-1}\end{array}$} & \multicolumn{2}{|c|}{ Number of experiments } \\
\hline & ${ }^{12} \mathrm{C}$ & ${ }^{13} \mathrm{C}$ & ${ }^{12} \mathrm{C}$ & ${ }^{13} \mathrm{C}$ \\
\hline \multirow[t]{2}{*}{$\mathrm{A} \beta_{40}$} & $1622.3 \pm 0.1$ & $1580.6 \pm 0.2$ & 3 & 5 \\
\hline & $1684.6 \pm 0.1$ & $1638.3 \pm 0.3$ & & \\
\hline \multirow[t]{2}{*}{$\mathrm{A} \beta_{42}$} & $1625.5 \pm 0.4$ & $1582.9 \pm 0.4$ & 6 & 3 \\
\hline & $1684.6 \pm 0.2$ & $1639.3 \pm 0.2$ & & \\
\hline
\end{tabular}

spectral regions of $\beta$-sheet absorption. Their band positions are listed in Table 1 . The band at $1685 \mathrm{~cm}^{-1}$ is an indicator of oligomers and often considered a marker band for antiparallel $\beta$-sheets as discussed previously. ${ }^{27,65}$ The absence of distinct bands in the non- $\beta$-sheet spectral regions indicate a large content of $\beta$-sheets. Indeed, a fit to the absorbance spectra revealed a contribution of $\beta$-sheet bands to the total absorbance in the amide I region of $28 \%$ for $A \beta_{40}$ and of $39 \%$ for $A \beta_{42}$. Since $\beta$-sheets absorb stronger in ${ }^{2} \mathrm{H}_{2} \mathrm{O}$ than unordered structures by a factor of $\sim 1.5,{ }^{75,76}$ the $\beta$-sheet content is estimated to $\sim 20 \%$ for $A \beta_{40}$ and to $\sim 25 \%$ for $A \beta_{42}$. The $\beta$-sheet content in structured $\mathrm{A} \beta$ oligomers might be higher than these values because of the presence of unstructured monomers and aggregates.

The spectra of $A \beta_{40}$ and $A \beta_{42}$ are similar in shape but the band positions of the main band for unlabeled and labeled $\mathrm{A} \beta_{42}$ are found at $2-3 \mathrm{~cm}^{-1}$ higher wavenumbers than for $\mathrm{A} \beta_{40}$, indicating different $\beta$-sheet structures. ${ }^{65}$ The spectra of the oligomers are similar to those of $\mathrm{A} \beta_{42}: \mathrm{A} \beta_{40}$ hetero-oligomers that we have published previously. ${ }^{65}$

The main band of the unlabeled oligomers at $1622-1625 \mathrm{~cm}^{-1}$ and that of the labeled oligomers at $1580-1583 \mathrm{~cm}^{-1}$ shift towards higher wavenumber upon admixture of peptides with a different isotope. In the following, we will focus on the ${ }^{13} \mathrm{C}$-band because the ${ }^{12} \mathrm{C}$-band is not clearly seen at low ${ }^{12} \mathrm{C}$ abundance and because it is overlapped by the high wavenumber band of the ${ }^{13} \mathrm{C}$-spectrum. The shift of the ${ }^{13} \mathrm{C}$-band position for both oligomers is shown in Fig. 5.

\section{Calculation strategy}

In order to get information on the internal structure of individual peptide molecules within the oligomers, we calculated the

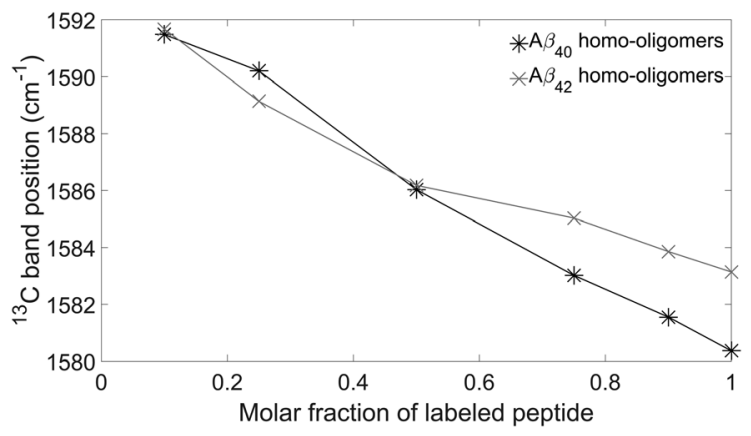

Fig. 5 Experimental ${ }^{13} \mathrm{C}$-band positions for $A \beta_{40}$ (black) and $A \beta_{42}$ (grey) oligomers. 
${ }^{13} \mathrm{C}$-band shift for a variety of oligomer model structures. The aim of these calculations was a quantitative agreement between calculated and experimental spectra. Here, the calculations faced two challenges: (i) the atomic structure of $\mathrm{A} \beta$ oligomers is unknown and (ii) the values for the parameters that enter the spectrum calculations are not known to the necessary precision. Regarding (i) we used a number of model structures including a published atomic model. Regarding (ii) we adjusted the calculation parameters to fit the experimental spectra of the entirely unlabeled and the entirely labeled samples as described in Computational methods. With these constraints, the spectra of the isotopic mixtures were calculated without further adjustments.

In our calculations, we considered different internal structures of the peptide molecules within the oligomers giving rise to different building blocks for the $\beta$-sheets (see Fig. 3). A building block in our calculations is the contribution of a single peptide molecule to the $\beta$-sheets of the oligomers. Within a given building block the carbon isotope is the same (with the exception of ${ }^{12} \mathrm{C}$ impurities in ${ }^{13} \mathrm{C}$ peptides, see Computational methods) and the vibrational coupling represents intramolecular coupling. Coupling between amide groups in different building blocks models the intermolecular coupling. We considered the following building blocks: a single strand, where the strand contributed by one peptide has neighboring strands from other peptides; a 2-strand block, where two adjacent strands contributed by one peptide molecule have 2-strand blocks from other peptides as neighbors; a 3-strand sheet, where each peptide monomer contributes three adjacent strands to the $\beta$-sheet of the oligomer. In addition, we considered two types of two non-adjacent strand arrangements: in interlaced model 1 , the two strands from one peptide molecule are separated by a strand from another peptide, while in interlaced model 2 the two strands are separated by two strands from two different peptides.

\section{General features of the calculated spectra}

Fig. 6 shows an example of an absorbance spectrum and its second derivative calculated by our program, using an ideal

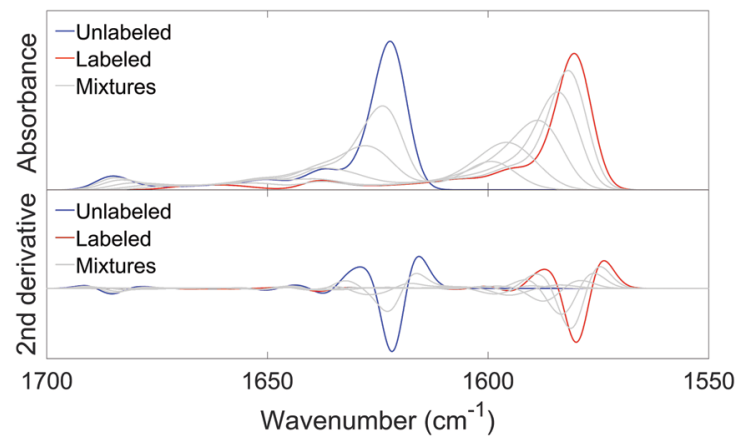

Fig. 6 Calculated absorbance and second derivative spectra for an antiparallel sheet with 12 strands and 10 residues per strand (9 complete amide groups), using the single strand building block. In the upper panel, the absorbance spectra are shown for the unlabeled structure (blue), the labeled structure (red) and mixtures $(9: 1,3: 1,1: 1,1: 3,1: 9)$ of unlabeled and labeled strands (grey). In the lower panel, the second derivatives of the absorbance spectra in the upper panel are shown, using the same color code. antiparallel sheet with 12 strands and 10 residues per strand (9 complete amide groups) and the single strand building block. Each line in Fig. 6 is the average of 20 repetitions of 3000 statistical compositions for each ${ }^{13} \mathrm{C}:{ }^{12} \mathrm{C}$ ratio. The blue line and the red line represent the two isotopically pure sheets. Each grey line represents a mixture of unlabeled and labeled species, using the same ${ }^{13} \mathrm{C}:{ }^{12} \mathrm{C}$ ratios as in the experiments.

As in the experimental spectra, the main ${ }^{12} \mathrm{C}$ - and ${ }^{13} \mathrm{C}$-bands shift down when the $\beta$-sheets become isotopically purer and the high wavenumber $\beta$-sheet band shifts up. We will focus on the shift of the main ${ }^{13} \mathrm{C}$-band in the $1600-1580 \mathrm{~cm}^{-1}$ range and use the term $0.1 \rightarrow 1{ }^{13} \mathrm{C}$-band shift for a downshift of the ${ }^{13} \mathrm{C}$-band position upon changing the ${ }^{13} \mathrm{C}:{ }^{12} \mathrm{C}$ ratio from 0.1 to 1 .

\section{The role of interstrand coupling}

The shift of the ${ }^{13} \mathrm{C}$-band upon ${ }^{13} \mathrm{C}$-enrichment is due to vibrational coupling between different strands of a $\beta$-sheet. Without interstrand coupling, an isolated (but hydrogen bonded) strand would absorb at the same wavenumber as an entire sheet. This is also true for a single ${ }^{13} \mathrm{C}$-strand surrounded by ${ }^{12} \mathrm{C}$-strands, which would absorb at the same wavenumber as an entirely labeled sheet. Therefore, in the absence of interstrand coupling the ${ }^{13} \mathrm{C}$-band shift would be zero.

Even in the presence of interstrand coupling, a ${ }^{13} \mathrm{C}$-strand that is surrounded by ${ }^{12} \mathrm{C}$-strands is rather uncoupled from the rest of the sheet. In test calculations of such a system with a $\beta$-sheet composed of 12 strands with 10 residues ( 9 complete amide groups) each and the labeled strand close to the middle of the sheet, only $\sim 15 \%$ of the vibrational energy of the two vibrations with highest intensity in the ${ }^{13} \mathrm{C}$ spectral region was contributed by ${ }^{12} \mathrm{C}$-amide groups. Accordingly, a single ${ }^{13} \mathrm{C}$-strand in an otherwise ${ }^{12} \mathrm{C}$-sheet absorbs similarly (1600 $\mathrm{cm}^{-1}$ in our calculation) to a ${ }^{13} \mathrm{C}$-strand that is entirely decoupled from the ${ }^{12} \mathrm{C}$-strands $\left(1605 \mathrm{~cm}^{-1}\right)$. The situation is similar at low ${ }^{13} \mathrm{C}:{ }^{12} \mathrm{C}$ ratios because the large distance between the ${ }^{13} \mathrm{C}$-strands prevents an effective coupling. When more ${ }^{13} \mathrm{C}$-strands are incorporated into the sheet, ${ }^{13} \mathrm{C}$-interstrandcoupling becomes noticeable because the ${ }^{13} \mathrm{C}$-strands get closer and this shifts the main $\beta$-sheet band to lower wavenumbers. ${ }^{45}$ In summary, the ${ }^{13} \mathrm{C}$-band shift is different from zero when ${ }^{13} \mathrm{C}$-strands are sufficiently close to enable effective coupling between amide I vibrations in different ${ }^{13} \mathrm{C}$-strands.

\section{Influence of $\beta$-sheet structure}

In the following, we discuss the calculations for $A \beta_{40}$. We used a set of model structures consisting of $7 \beta$-sheets (4-24 strands) and $11 \beta$-barrels (8-22 strands). For each of the model structures, the parameters were adjusted so that the calculated spectra matched the experimental spectrum of the unlabeled sample. Then the spectra of the isotope mixtures were calculated and the ${ }^{13} \mathrm{C}$-band shifts at a certain molar ratio of labeled peptides averaged for all structures.

The shifts calculated for $\beta$-barrels of various sizes are rather independent of the number of strands in the barrel, whereas those calculated for flat antiparallel $\beta$-sheets increase with the number of strands in the sheet, in particular for small sheets 
(see Table S4, ESI $\dagger$ ). When the building block is a single strand, the steepest dependency is between two and six strands, whereas for building blocks of two and three strands, considerable sensitivity is observed up to 12 strands.

In contrast, the number of residues per strand has a minor role: reducing it from 10 residues ( 9 complete amide groups) to 6 residues or 4 residues, decreases the ${ }^{13} \mathrm{C}$-band shift by at most $0.5 \mathrm{~cm}^{-1}$ using the strand building block and sheets with 6 and 12 strands (see Table S4, ESI $\dagger$ ).

A further influence that we considered is that of intra-chain coupling where the dominant contribution is the interaction between the nearest neighbors. This interaction depends on the backbone dihedral angles and can be smaller or larger than for ideal antiparallel $\beta$-sheets when the dihedral angles deviate from the ideal values. ${ }^{48-51}$ In the context of the evaluation of our dodecamer results (see below), we tested the effect of reducing the intra-chain nearest neighbor coupling to $1 / 3$ of the original value. This required us to increase the magnitude of the dipole derivative in order to match the splitting of the $\beta$-sheet bands of experiment and calculation. This led to stronger interstrand coupling and thus to a larger ${ }^{13} \mathrm{C}$-band shift $\left(4 \mathrm{~cm}^{-1}\right.$ larger for a 12 -stranded sheet and the single strand building block).

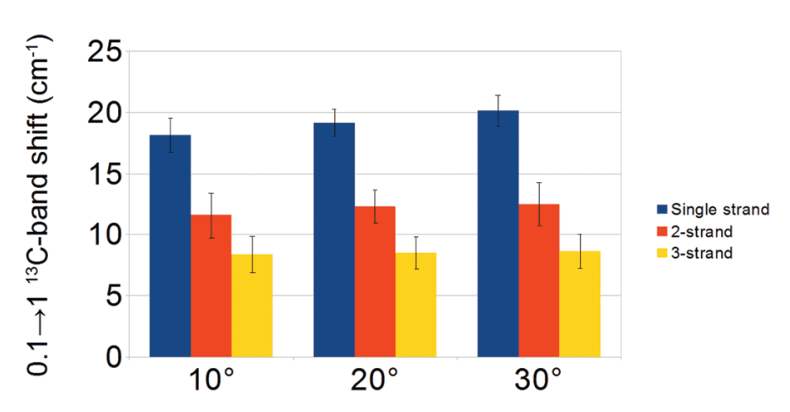

Angle of dipole derivative

Fig. $7 \quad 0.1 \rightarrow 1{ }^{13} \mathrm{C}$-band shifts, averaged from sets of four $\beta$-sheets and four $\beta$-barrels, using different angles of the dipole derivative. The calculation parameters were adjusted to match the all-unlabeled experimental spectrum for $A \beta_{40}$. Because of the adjustment, the magnitude of the dipole derivative had to be increased when we increased the angle of the dipole derivative. A higher magnitude led to a slightly stronger coupling between the strands, so the shifts of the ${ }^{13} \mathrm{C}$-band position for the three building blocks are somewhat larger for larger angles.

\section{Influence of the dipole derivative angle}

The angle of the dipole derivative with respect to the $\mathrm{C}=\mathrm{O}$ bond is a parameter that could not be adjusted from a comparison with experiment. Instead, we used fixed values of $10^{\circ}, 20^{\circ}$, and $30^{\circ}$ for a sub-set of four sheets $(4,6,12$ and 24 strands) and four barrels (8, 12, 18 and 22 strands). Similar ${ }^{13} \mathrm{C}$-band shifts were found for these angles, which are shown in Fig. 7 and tabulated in Table 2. They demonstrate that the calculation results are robust against a change in dipole derivative angle. Therefore, we will discuss only the results for an angle of $20^{\circ}$ in the following.

\section{A $\boldsymbol{\beta}_{40}$ oligomers}

Fig. 8 shows the average ${ }^{13} \mathrm{C}$-band shifts obtained for the $\mathrm{A} \beta_{40}$ calculations and Table 2 collates the $0.1 \rightarrow 1{ }^{13} \mathrm{C}$-band shifts.

The figure shows that the ${ }^{13} \mathrm{C}$-band shift depends on the building block that each peptide monomer contributes to the oligomer structure. The shifts at $10 \%$ and $25 \%{ }^{13} \mathrm{C}$-peptide content are largest for the strand building block, smaller for the 2-strand building block and even smaller for the 3-strand building block.

For a particular building block, the standard deviation for all model structures is smaller than the difference between

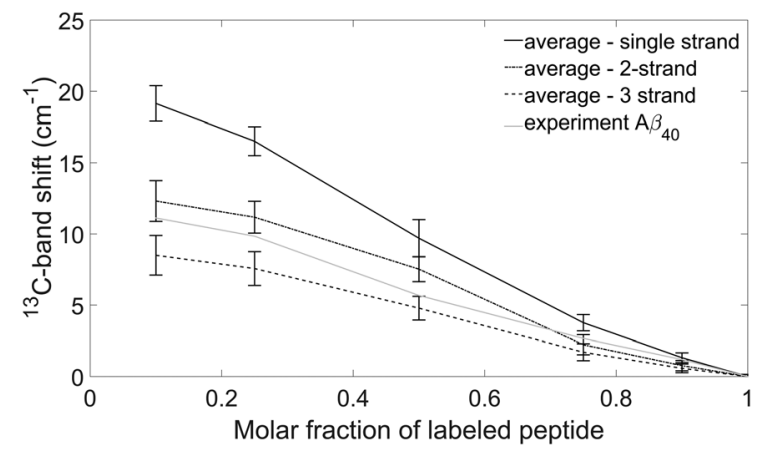

Fig. 8 Experimental and simulated ${ }^{13} \mathrm{C}$-band shifts for $A \beta_{40}$ oligomers. The calculations were done for 18 model structures (see text) with a dipole derivative angle of $20^{\circ}$ and the obtained band shifts averaged. The black solid line is the average ${ }^{13} \mathrm{C}$-band shift using the single strand building block. The dash-dot line is the average ${ }^{13} \mathrm{C}$-band shift using the 2 -strand building block. The dashed line is the average ${ }^{13} \mathrm{C}$-band shift using the 3 -strand building block. The grey solid line shows the experimental ${ }^{13} \mathrm{C}$ band shift. The error bars indicate the standard deviations of the band positions calculated for the different model structures.

Table 2 Resume of the listed experimental and calculated $0.1 \rightarrow 1{ }^{13} \mathrm{C}$-band shifts. The term $0.1 \rightarrow 1{ }^{13} \mathrm{C}$-band shift indicates a shift of the position of the ${ }^{13} \mathrm{C}$-band in the $1600-1580 \mathrm{~cm}^{-1}$ spectral range upon changing the ${ }^{13} \mathrm{C}:{ }^{12} \mathrm{C}$ ratio from 0.1 to 1

\begin{tabular}{|c|c|c|c|c|c|c|}
\hline & & \multicolumn{5}{|c|}{$\underline{0.1 \rightarrow 1{ }^{13} \mathrm{C} \text {-band shift }\left(\text { in } \mathrm{cm}^{-1}\right) \pm \text { standard deviation }}$} \\
\hline & & Single strand & 2-strand block & 3-strand block & Interlaced model 1 & Interlaced model 2 \\
\hline \multirow[t]{5}{*}{$\mathrm{A} \beta_{40}$} & Experiment & 11.1 & & & & \\
\hline & Angle: $10^{\circ}$ & $18.2 \pm 1.4$ & $11.6 \pm 1.8$ & $8.4 \pm 1.5$ & & \\
\hline & Angle: $20^{\circ}$ & $19.2 \pm 1.1$ & $12.3 \pm 1.3$ & $8.5 \pm 1.3$ & $15.5 \pm 1.8$ & $17.3 \pm 1.3$ \\
\hline & Angle: $30^{\circ}$ & $20.1 \pm 1.3$ & $12.5 \pm 1.8$ & $8.6 \pm 1.4$ & & \\
\hline & Angle: $20^{\circ}$ - dodecamer & & 12.5 & & & \\
\hline \multirow[t]{3}{*}{$\mathrm{A} \beta_{42}$} & Experiment & 8.5 & & & & \\
\hline & Angle: $20^{\circ}$ - sheet & 17.1 & 11.5 & 8.2 & & \\
\hline & Angle: $20^{\circ}$ - barrel & 18.6 & 11.7 & 7.7 & & \\
\hline
\end{tabular}


different building blocks (see Fig. 8). Accordingly, the ${ }^{13} \mathrm{C}$-band shift is more sensitive to the nature of the building block than to the particular structure used for the calculation. This demonstrates that the ${ }^{13} \mathrm{C}$-band shift can be used to study the internal structure of individual peptides in $\mathrm{A} \beta$ oligomers.

The ${ }^{13} \mathrm{C}$-band shifts for the interlaced building blocks are in between the shifts for the single strand and the 2-strand building blocks. They are listed in Table 2 , but not shown in Fig. 8. The results can be explained at the limiting case of highly diluted labeled peptide. Then the neighboring peptides will be unlabeled and the insertion of one or two unlabeled strands between the two strands of the labeled peptide weakens the coupling between the labeled strands, which leads to a higher ${ }^{13} \mathrm{C}$-band position than in the case of two adjacent strands.

The experimental ${ }^{13} \mathrm{C}$-band shift is shown as grey line in Fig. 8. The $0.1 \rightarrow 1{ }^{13} \mathrm{C}$-band shift is $11.1 \mathrm{~cm}^{-1}$ which is very close to the average shift calculated for our model structures and the 2 -strand building block $\left(12.3 \mathrm{~cm}^{-1}\right)$. This shift depends to some degree on the structure of the $\beta$-sheets (see section Influence of $\beta$-sheet structure), which is reflected in the error bars in Fig. 8. For example, when a sheet consists of only two strands, the calculated $0.1 \rightarrow 1{ }^{13} \mathrm{C}$-band shift for the single strand building block is $12.6 \mathrm{~cm}^{-1}$, which is close to the shift observed experimentally for $A \beta_{40}$. However, such a small sheet with only two strands can be excluded from the experimental position of the main $\beta$-sheet band as further discussed below (see section Structural constraints from experiments and calculations for $A \beta$ oligomers). In conclusion, our results indicate that each $\mathrm{A} \beta_{40}$ molecule contributes two adjacent strands to the $\beta$-sheets of the oligomers.

In addition to our model structures, we investigated a structure obtained by X-ray crystallography of a modified $A \beta_{17-36}$ peptide. The structure of this peptide has been stabilized by introducing a disulfide bridge between residues 24 and 29 and by linking the $\mathrm{N}$ - and $\mathrm{C}$-terminus via an ornithine molecule. In the X-ray structure (pdb entry $5 \mathrm{HOW}^{23}$ ), each peptide forms a $\beta$-hairpin and assembles with two other peptides in a triangular unit that consists of distorted 4 -stranded $\beta$-sheets at both ends of the hairpins. Four triangular units assemble to a dodecamer, which was used in our calculations. Because of the $\beta$-hairpin structure of individual peptides, this structure corresponds to our 2-strand building block. In our calculation the $0.1 \rightarrow 1{ }^{13} \mathrm{C}$-band shift is $12.5 \mathrm{~cm}^{-1}$. It is close to the average shift calculated with the 2-strand building block for our model structures $\left(12.3 \mathrm{~cm}^{-1}\right)$. This shift seems to be caused by two opposing effects. (i) The small number of strands in the sheet of the triangular unit is expected to cause a smaller ${ }^{13} \mathrm{C}$-band shift. An even smaller shift of only $8.3 \mathrm{~cm}^{-1}$ was calculated for a model sheet with four strands and the 2-strand building block (Table S4, ESI $\dagger$ ). (ii) The deviation of the dihedral angles from those of an ideal antiparallel $\beta$-sheet is expected to increase the ${ }^{13} \mathrm{C}$-band shift. An inspection of the Ramachandran plot of the structure revealed that many dihedral backbone angles deviate from those of an ideal antiparallel sheet. The average angles were
$\Phi=-98.5^{\circ}$ and $\Psi=113.4^{\circ}$ (the dihedral angles of our ideal antiparallel $\beta$-sheets were $-138.6^{\circ}$ and $134.5^{\circ}$, respectively). These angles lead to a weaker nearest neighbor coupling ${ }^{48}$ which generates a larger ${ }^{13} \mathrm{C}$-band shift with our approach as discussed above in section Influence of $\beta$-sheet structure. Taken together, the ${ }^{13} \mathrm{C}$-band shift calculated for the dodecamer structure can be explained by the small number of strands in the sheets and a smaller nearest neighbor coupling than in ideal sheets.

We note that the calculated all-unlabeled spectrum of the dodecamer contains two bands at 1622 and $1639 \mathrm{~cm}^{-1}$ in the main region of $\beta$-sheet absorption. The higher wavenumber band is not observed in our experiments.

Summarizing the results for the $0.1 \rightarrow 1{ }^{13} \mathrm{C}$-band shift of $A \beta_{40}$ oligomers, the experimental shift is close to that calculated for the dodecamer and to the average shift of $\beta$-sheets and $\beta$-barrels when the building block consists of two adjacent strands. We conclude that our experimental isotope shift for $\mathrm{A} \beta_{40}$ oligomers is well explained by $\beta$-sheet structures in which individual $A \beta_{40}$ peptides adopt a $\beta$-hairpin structure.

\section{$A \beta_{42}$ oligomers}

In $A \beta_{42}$ oligomers, the experimental $0.1 \rightarrow 1{ }^{13} \mathrm{C}$-band shift is $8.5 \mathrm{~cm}^{-1}$, which is less than for $A \beta_{40}\left(11.1 \mathrm{~cm}^{-1}\right)$. For our $A \beta_{42}$ calculations, we considered only one $\beta$-sheet and one $\beta$-barrel, both with 12 strands. These particular structures were chosen because they generate ${ }^{13} \mathrm{C}$-band shifts close to the average shifts in the $A \beta_{40}$ calculations shown in Fig. 8. Their calculated ${ }^{12} \mathrm{C}$-spectra were matched to the experimental spectrum and the ${ }^{13} \mathrm{C}$-band positions of isotope mixtures are shown in Fig. 9 for the three building blocks. Table 2 lists the ${ }^{13} \mathrm{C}$-band shifts obtained. As shown in Fig. 9, the experimental ${ }^{13} \mathrm{C}$-band shift matches closely the calculated shift for the 3-strand model. This might indicate that individual $A \beta_{42}$ peptides contribute more than two adjacent strands to the $\beta$-sheets of the oligomers. We consider this conclusion to be valid for sheets of moderate size (12 and more strands). However, when the number of strands per sheet is smaller, the ${ }^{13} \mathrm{C}$-band shift is also smaller

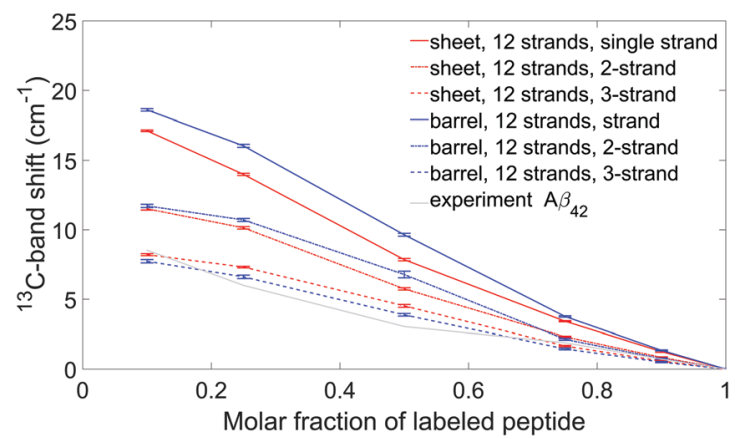

Fig. 9 Experimental and simulated ${ }^{13} \mathrm{C}$-band shifts for $A \beta_{42}$ oligomers for two specific models: a sheet and a barrel, each composed of 12 strands. The dipole derivative angle was $20^{\circ}$. Each model is represented by a different color, while the three building blocks are shown using three different line style (as in Fig. 8). Experimental band positions are shown using a grey solid line. 
and is similar to the experimental shift for a 4-stranded sheet calculated with the 2-strand building block. Therefore, we cannot exclude that the building block consists of only two strands. We conclude that the $\beta$-sheets of $A \beta_{42}$ either contain a small number of strands, where each peptide molecule contributes a $\beta$-hairpin, or contain a larger number of strands, where each peptide contributes more than two adjacent strands to the $\beta$-sheets.

\section{Discussion}

\section{Comparison with previous infrared studies}

We studied the effects of the isotope composition of $\beta$-sheets on the amide I infrared spectrum. We focused on the ${ }^{13} \mathrm{C}$-band shift in order to elucidate the internal structure of $\mathrm{A} \beta$ peptides in $A \beta_{42}$ and $A \beta_{40}$ oligomers. For all model structures investigated, the shift depends on the structural unit (building block) that each individual peptide molecule contributes to the structure of the oligomers. The differences between the shifts of different building blocks were larger than the standard deviations calculated from the results for different model structures and therefore are useful for structural studies. This is particularly true for the most diluted cases $\left(0.1\right.$ and $0.25{ }^{13} \mathrm{C}:{ }^{12} \mathrm{C}$-ratio) which highlights their importance for the analysis. ${ }^{56}$

Related to this work, Matos et al. ${ }^{30}$ investigated mixtures of $\mathrm{A} \beta_{42}$ and pyroglutamylated $\mathrm{A} \beta_{3-42}$. Upon admixture of $50 \%$ pyroglutamylated ${ }^{12} \mathrm{C}-\mathrm{A} \beta_{3-42}$ to $\mathrm{A} \beta_{42}$, they observed an upshift of the ${ }^{13} \mathrm{C}-\mathrm{A} \beta_{42}$ band from $1585 \mathrm{~cm}^{-1}$ to $1595 \mathrm{~cm}^{-1}$. This is much larger than the shift of $3 \mathrm{~cm}^{-1}$ for the corresponding isotope dilution step for our $A \beta_{42}$ oligomers, and of $6 \mathrm{~cm}^{-1}$ for our $A \beta_{40}$ oligomers. Therefore, the structure of an $A \beta_{42}$ peptide in the aggregates with pyroglutamylated $A \beta_{3-42}$ seems to be different from that in our $A \beta_{40}$ and $A \beta_{42}$ homo-oligomers as well as in our $A \beta_{40}: A \beta_{42}$ hetero-oligomers. The $10 \mathrm{~cm}^{-1}$ shift observed by Matos et al. is slightly larger than the average shifts for the single strand building block calculated for our model structures (Fig. 8 and 9). Therefore, individual $A \beta_{42}$ molecules likely contribute only a single strand to the aggregates with pyroglutamylated $\mathrm{A} \beta_{3-42}$.

Moran et $a l .{ }^{56}$ investigated amyloid fibrils formed by the eye lens protein $\gamma$ D-crystallin in ${ }^{2} \mathrm{H}_{2} \mathrm{O}$. Their $0.1 \rightarrow 1{ }^{13} \mathrm{C}$-band shift of $22 \mathrm{~cm}^{-1}$ was judged to be consistent with contributions of either one or two strands per protein. ${ }^{56}$ An even larger shift of nearly $30 \mathrm{~cm}^{-1}$ was observed for thermally induced aggregates, which is consistent with a contribution of one strand per protein to the $\beta$-sheets of the aggregates. ${ }^{77}$ We note that both the unlabeled and labeled $\gamma \mathrm{D}$-crystallin aggregates exhibit lower band positions than our $\mathrm{A} \beta$ samples, which seems to indicate stronger interstrand coupling and thus explains that Moran et al. observe larger shifts than we calculate.

From a similar isotope dilution experiment, Buchanan et $a l .{ }^{59}$ concluded that each monomer within their antiparallel polyQ amyloid fibrils contributes two adjacent strands. Their experimental $0.1 \rightarrow 1{ }^{13} \mathrm{C}$-band shift of $12 \mathrm{~cm}^{-1}$ is close to our average shifts for the 2-strand block $\left(11.6 \mathrm{~cm}^{-1}, 12.3 \mathrm{~cm}^{-1}\right.$ and
$12.5 \mathrm{~cm}^{-1}$ for the $\mathrm{A} \beta_{40}$ calculations and dipole derivative angles of $10^{\circ}, 20^{\circ}$, and $30^{\circ}$, respectively) demonstrating agreement between the two studies.

\section{Structural constraints from experiments and calculations for A $\beta$ oligomers}

In the present study, we analyzed the ${ }^{13} \mathrm{C}$-band shift of $\mathrm{A} \beta$ oligomers that contained a mixture of labeled and unlabeled peptides. Because the structure of $A \beta$ oligomers is debated, we used a large number of model structures for our analysis. We also matched the calculations to the experimental spectra. This approach removed two biases - those of selecting a particular structure and particular calculation parameters. This made possible a direct quantitative comparison between experiment and calculations. It turned out that the results were robust in the sense that different internal structures of $\mathrm{A} \beta$ produce quantitatively different shifts irrespective of the particular overall $\beta$-sheet structure used for the calculations. For further improvement of the calculations, more information about the structure of $\mathrm{A} \beta$ oligomers is needed.

According to the results of our study, $\mathrm{A} \beta$ molecules contribute more than just a single strand to the structure of the oligomers. Also the two interlaced building blocks, the ${ }^{13} \mathrm{C}$-band shifts of which are located between those of the single strand block and of the 2-strand block, failed to reproduce the experimentally observed shifts. Instead, our experiments with $\mathrm{A} \beta_{40}$ are best described by a contribution of two adjacent strands from each peptide molecule. For $A \beta_{42}$, contributions of two or more adjacent strands are possible or a combination of these. These structural constraints are fulfilled by (successive) $\beta$-hairpins, which are also in line with the antiparallel orientation of the $\beta$-strands inferred from the observation of a distinct high wavenumber band.

A further structural constraint can be derived from the band position of the main $\beta$-sheet band and the splitting between high and low wavenumber band, which are sensitive to the number of strands in a sheet. ${ }^{45,78}$ For the all-unlabeled samples it is found below $1626 \mathrm{~cm}^{-1}$ for both peptides and the splitting is $\sim 60 \mathrm{~cm}^{-1}$. Designed $\beta$-hairpins and 3-stranded antiparallel sheets absorb at a higher wavenumber, near $\sim 1635 \mathrm{~cm}^{-1}$ in ${ }^{2} \mathrm{H}_{2} \mathrm{O}$, and the splitting is smaller: $\sim 40 \mathrm{~cm}^{-1} \cdot{ }^{79-81}$ From this comparison we deduce that the $\beta$-sheets of our $A \beta$ oligomers are formed by at least four strands.

We conclude that our experimental spectra and ${ }^{13} \mathrm{C}$-band shifts can be well explained by flat, antiparallel $\beta$-sheets or by $\beta$-barrels with at least four strands in which each peptide molecule contributes two adjacent strands to $A \beta_{40}$ oligomers and two or more adjacent strands to $A \beta_{42}$ oligomers. Less uniform structures than flat sheets and barrels, like that of the dodecamer, ${ }^{23}$ are likely to produce more distinct bands than the two bands that we observed.

In our previous study, ${ }^{65}$ we studied $\mathrm{A} \beta_{40}: \mathrm{A} \beta_{42}$ heterooligomers in which one of the peptides was labeled. A comparison with homo-oligomers (see Fig. 2 of that publication) shows very similar shifts of the ${ }^{13} \mathrm{C}$-band upon isotope enrichment which we regard identical within experimental error. This indicates that 
the structure of each peptide is similar in homo- and heterooligomers.

Our structural constraints are consistent with oligomer models that propose a hairpin structure for individual peptide molecules. ${ }^{17,23,24,82}$ They are also in line with the suggestion that $\mathrm{A} \beta_{42}$ may fold into units with more strands per molecule. ${ }^{21}$ Models in which a considerable section of the peptide contributes only one strand to the oligomers ${ }^{16,18}$ do not well explain the isotope effect seen for our oligomers. The hairpins should be organized in a $\beta$-sheet with at least four strands or in a $\beta$-barrel. The latter has been suggested previously ${ }^{24}$ on the basis of the infrared absorption spectrum. Sheets and barrels with little structural distortions account well for our data. The trends seen in our results can be generalized also to other aggregating peptides. Those aggregates with amide I band positions similar to ours will produce ${ }^{13} \mathrm{C}$-band shifts that can be compared quantitatively to our calculations. This enables to deduce the internal structure of individual peptide molecules in these aggregates.

\section{Conflicts of interest}

There are no conflicts to declare.

\section{Acknowledgements}

We are grateful to Eeva-Liisa Karjalainen for the original version of the Matlab program for spectrum calculations and to Erik Goormaghtigh (Université Libre de Bruxelles) for providing the program Kinetics. We acknowledge support from Stockholms läns landsting, Wenner-Gren Stiftelserna, Knut och Alice Wallenbergs Stiftelse, Magnus Bergvalls Stiftelse and Stiftelsen Lars Hiertas Minne.

\section{References}

1 D. R. Thal, J. Walter, T. C. Saido and M. Fändrich, Acta Neuropathol., 2015, 129, 167-182.

2 R. Aleksis, F. Oleskovs, K. Jaudzems, J. Pahnke and H. Biverstål, Biochimie, 2017, 140, 176-192.

3 F. Chiti and C. M. Dobson, Annu. Rev. Biochem., 2017, 86, 27-68.

4 I. Benilova, E. Karran and B. De Strooper, Nat. Neurosci., 2012, 15, 349-357.

5 J. A. Hardy and G. A. Higgins, Science, 1992, 256, 184-185.

6 J. Hardy and D. Allsop, Trends Pharmacol. Sci., 1991, 12, 383-388.

7 D. J. Selkoe, Neuron, 1991, 6, 487-498.

8 K. Beyreuther and C. L. Masters, Brain Pathol., 1991, 1, 241-251.

9 D. J. Selkoe and J. Hardy, EMBO Mol. Med., 2016, 8, 595-608.

10 B. Caughey and P. T. Lansbury, Annu. Rev. Neurosci., 2003, 26, 267-298.

11 K. L. Viola and W. L. Klein, Acta Neuropathol., 2015, 129, 183-206.
12 C. Haass and D. J. Selkoe, Nat. Rev. Mol. Cell Biol., 2007, 8, 101-112.

13 E. Y. Hayden and D. B. Teplow, Alzheimer's Res. Ther., 2013, $5,60$.

14 S. Lesné, Swiss Med. Wkly., 2014, 144, w14021.

15 M. Fändrich, J. Mol. Biol., 2012, 421, 427-440.

16 L. Yu, R. Edalji, J. E. Harlan, T. F. Holzman, A. P. Lopez, B. Labkovsky, H. Hillen, S. Barghorn, U. Ebert, P. L. Richardson, L. Miesbauer, L. Solomon, D. Bartley, K. Walter, R. W. Johnson, P. J. Hajduk and E. T. Olejniczak, Biochemistry, 2009, 48, 1870-1877.

17 C. Lendel, M. Bjerring, A. Dubnovitsky, R. T. Kelly, A. Filippov, O. N. Antzutkin, N. C. Nielsen and T. Härd, Angew. Chem., Int. Ed., 2014, 53, 12756-12760.

18 W. M. Tay, D. Huang, T. L. Rosenberry and A. K. Paravastu, J. Mol. Biol., 2013, 425, 2494-2508.

19 D. Huang, M. I. Zimmerman, P. K. Martin, A. J. Nix, T. L. Rosenberry and A. K. Paravastu, J. Mol. Biol., 2015, 427, 2319-2328.

20 S. Chimon, M. A. Shaibat, C. R. Jones, D. C. Calero, B. Aizezi and Y. Ishii, Nat. Struct. Mol. Biol., 2007, 14, 1157-1164.

21 M. Ahmed, J. Davis, D. Aucoin, T. Sato, S. Ahuja, S. Aimoto, J. I. Elliott, W. E. Van Nostrand and S. O. Smith, Nat. Struct. Mol. Biol., 2010, 17, 561-567.

22 V. A. Streltsov, J. N. Varghese, C. L. Masters and S. D. Nuttall, J. Neurosci., 2011, 31, 1419-1426.

23 A. G. Kreutzer, I. L. Hamza, R. K. Spencer and J. S. Nowick, J. Am. Chem. Soc., 2016, 138, 4634-4642.

24 E. Cerf, R. Sarroukh, S. Tamamizu-Kato, L. Breydo, S. Derclaye, Y. F. Dufrêne, V. Narayanaswami, E. Goormaghtigh, J.-M. Ruysschaert and V. Raussens, Biochem. J., 2009, 421, 415-423.

25 B. Martial, T. Lefèvre and M. Auger, Biophys. Rev., 2018, 10, 1133-1149.

26 H. Komatsu, L. Liu, I. V. J. Murray and P. H. Axelsen, Biochim. Biophys. Acta, Biomembr., 2007, 1768, 1913-1922.

27 R. Sarroukh, E. Goormaghtigh, J.-M. Ruysschaert and V. Raussens, Biochim. Biophys. Acta, Biomembr., 2013, 1828, 2328-2338.

28 K. Nunez, J. Kay, A. Krotow, M. Tong, A. R. Agarwal, E. Cadenas and S. M. de la Monte, J. Alzheimer's Dis., 2016, 51, 151-163.

29 J. Nguyen, M. A. Baldwin, F. E. Cohen and S. B. Prusiner, Biochemistry, 1995, 34, 4186-4192.

30 J. O. Matos, G. Goldblatt, J. Jeon, B. Chen and S. A. Tatulian, J. Phys. Chem. B, 2014, 118, 5637-5643.

31 S. D. Moran and M. T. Zanni, J. Phys. Chem. Lett., 2014, 5, 1984-1993.

32 S. M. Decatur, Acc. Chem. Res., 2006, 39, 169-175.

33 Z. Ganim, H. S. Chung, A. W. Smith, L. P. DeFlores, K. C. Jones and A. Tokmakoff, Acc. Chem. Res., 2008, 41, 432-441.

34 Y. S. Kim and R. M. Hochstrasser, J. Phys. Chem. B, 2009, 113, 8231-8251.

35 J. Manor and I. T. Arkin, Biochim. Biophys. Acta, Biomembr., 2013, 1828, 2256-2264. 
36 J. Ma, I. M. Pazos, W. Zhang, R. M. Culik and F. Gai, Annu. Rev. Phys. Chem., 2015, 66, 357-377.

37 H. Torii and M. Tasumi, J. Chem. Phys., 1992, 96, 3379-3387.

$38 \mathrm{H}$. Torii and M. Tasumi, in Infrared spectroscopy of biomolecules, ed. H. H. Mantsch and D. Chapman, Wiley-Liss, New York, 1996, pp. 1-18.

39 A. Amadei, I. Daidone, A. Di Nola and M. Aschi, Curr. Opin. Struct. Biol., 2010, 20, 155-161.

40 J. Jeon, S. Yang, J.-H. Choi and M. Cho, Acc. Chem. Res., 2009, 42, 1280-1289.

41 T. la Cour Jansen, J. Knoester and C. Jansen, J. Chem. Phys., 2006, 124, 44502.

42 H. Torii, T. Tatsumi and M. Tasumi, J. Raman Spectrosc., 1998, 29, 537-546.

43 H. Torii, T. Tatsumi, T. Kanazawa and M. Tasumi, J. Phys. Chem. B, 1998, 102, 309-314.

44 S. Ham, J.-H. Kim, H. Lee and M. Cho, J. Chem. Phys., 2003, 118, 3491-3498.

45 Y. N. Chirgadze and N. A. Nevskaya, Biopolymers, 1976, 15, 607-625.

46 S. Krimm and Y. Abe, Proc. Natl. Acad. Sci. U. S. A., 1972, 69, 2788-2792.

47 T. C. Cheam and S. Krimm, Chem. Phys. Lett., 1984, 107, 613-616.

48 T. la Cour Jansen, A. G. Dijkstra, T. M. Watson, J. D. Hirst and J. Knoester, J. Chem. Phys., 2006, 125, 44312.

49 T. la Cour Jansen, A. G. Dijkstra, T. M. Watson, J. D. Hirst and J. Knoester, J. Chem. Phys., 2012, 136, 209901.

50 H. Torii and M. Tasumi, J. Raman Spectrosc., 1998, 229, 81-86.

51 S. Ham and M. Cho, J. Chem. Phys., 2003, 118, 6915-6922.

52 J.-H. Choi and M. Cho, J. Chem. Phys., 2004, 120, 4383-4392.

53 R. D. Gorbunov, D. S. Kosov and G. Stock, J. Chem. Phys., 2005, 122, 224904.

54 J.-H. Choi, S. Ham and M. Cho, J. Chem. Phys., 2002, 117, 6821.

55 J. P. Lomont, K. L. Rich, M. Maj, J.-J. Ho, J. S. Ostrander and M. T. Zanni, J. Phys. Chem. B, 2018, 122, 144-153.

56 S. D. Moran, A. M. Woys, L. E. Buchanan, E. Bixby, S. M. Decatur and M. T. Zanni, Proc. Natl. Acad. Sci. U. S. A., 2012, 109, 3329-3334.

57 Z. Ganim and A. Tokmakoff, Biophys. J., 2006, 91, 2636-2646.

58 T. M. Watson and J. D. Hirst, Phys. Chem. Chem. Phys., 2004, 6, 998-1005.

59 L. E. Buchanan, J. K. Carr, A. M. Fluitt, A. J. Hoganson, S. D. Moran, J. J. de Pablo, J. L. Skinner and M. T. Zanni, Proc. Natl. Acad. Sci. U. S. A., 2014, 111, 5796-5801.
60 S. Hahn, S. Ham and M. Cho, J. Phys. Chem. B, 2005, 109, 11789-11801.

61 L. Wang, C. T. Middleton, M. T. Zanni and J. L. Skinner, Methods, 2011, 3713-3724.

62 E. Małolepsza and J. E. Straub, J. Phys. Chem. B, 2014, 118, 7848-7855.

63 H. Torii, J. Phys. Chem. B, 2007, 111, 5434-5444.

64 Y. Abe and S. Krimm, Biopolymers, 1972, 11, 1817-1839.

65 M. Baldassarre, C. M. Baronio, L. A. Morozova-Roche and A. Barth, Chem. Sci., 2017, 8, 8247-8254.

66 M. Baldassarre, C. Li, N. Eremina, E. Goormaghtigh and A. Barth, Molecules, 2015, 20, 12599-12622.

67 E.-L. Karjalainen, T. Ersmark and A. Barth, J. Phys. Chem. B, 2012, 116, 4831-4842.

68 R. Fraser and T. MacRae, in Conformation in fibrous proteins and related synthetic polypeptides, ed. R. Fraser, Academic Press, New York, 1973, pp. 218-246.

69 I. Bertini, L. Gonnelli, C. Luchinat, J. Mao and A. Nesi, J. Am. Chem. Soc., 2011, 133, 16013-16022.

70 A. T. Petkova, Y. Ishii, J. J. Balbach, O. N. Antzutkin, R. D. Leapman, F. Delaglio and R. Tycko, Proc. Natl. Acad. Sci. U. S. A., 2002, 99, 16742-16747.

71 T. Lührs, C. Ritter, M. Adrian, D. Riek-Loher, B. Bohrmann, H. Döbeli, D. Schubert and R. Riek, Proc. Natl. Acad. Sci. U. S. A., 2005, 102, 17342-17347.

72 N. Guex and M. C. Peitsch, Electrophoresis, 1997, 18, 2714-2723. 73 W. H. Moore and S. Krimm, Biopolymers, 1976, 15, 2439-2464.

74 G. Cuevas, V. Renugopalakrishnan, G. Madrid and A. T. Hagler, Phys. Chem. Chem. Phys., 2002, 4, 1490-1499.

75 Y. N. Chirgadze, B. V. Shestopalov and S. Y. Venyaminov, Biopolymers, 1973, 12, 1337-1351.

76 M. Jackson, P. I. Haris and D. Chapman, Biochim. Biophys. Acta, Protein Struct. Mol. Enzymol., 1989, 998, 75-79.

77 S. D. Moran, T. O. Zhang and M. T. Zanni, Protein Sci., 2014, 23, 321-331.

78 J. Kubelka and T. A. Keiderling, J. Am. Chem. Soc., 2001, 123, 12048-12058.

79 J. Hilario, J. Kubelka and T. A. Keiderling, J. Am. Chem. Soc., 2003, 125, 7562-7574.

80 D. Scheerer, H. Chi, D. McElheny, T. A. Keiderling and K. Hauser, J. Phys. Chem. B, 2018, 122, 10445-10454.

81 Y. Xu, P. Purkayastha and F. Gai, J. Am. Chem. Soc., 2006, 128, 15836-15842.

82 A. Sandberg, L. M. Luheshi, S. Söllvander, T. Pereira de Barros, B. Macao, T. P. J. Knowles, H. Biverstål, C. Lendel, F. Ekholm-Petterson, A. Dubnovitsky, L. Lannfelt, C. M. Dobson and T. Härd, Proc. Natl. Acad. Sci. U. S. A., 2010, 107, 15595-15600. 